\title{
Performance Comparison of Different Advanced Control Schemes for Glucose Level Control under Disturbing Meal
}

\author{
Bashar Fateh Midhat* Amjad Jaleel Humaidi** \\ *,**Department of Control and Systems Engineering / University of Technology \\ *Email: basharfm88@yahoo.com \\ **Email: aaaacontrol2010@yahoo.com
}

(Received 30 October 2016; accepted 7 March 2017)

https://doi.org/10.22153/kej.2017.03.003

\begin{abstract}
In this work, diabetic glucose concentration level control under disturbing meal has been controlled using two set of advanced controllers. The first set is sliding mode controllers (classical and integral) and the second set is represented by optimal LQR controllers (classical and Min-, ax). Due to their characteristic features of disturbance rejection, both integral sliding mode controller and LQR Minmax controller are dedicated here for comparison. The Bergman minimal mathematical model was used to represent the dynamic behavior of a diabetic patient's blood glucose concentration to the insulin injection. Simulations based on Matlab/Simulink, were performed to verify the performance of each controller. In spite that Min-max optimal controller gave better disturbance rejection capability than classical optimal controller, classical sliding mode controller could outperform Min-max controller. However, it has been shown that integral sliding mode controller is the best of all in terms of disturbance rejection capability.
\end{abstract}

Key words: Optimal LQR control, Optimalminimax control, Sliding mode control, Integral sliding mode control.

\section{Introduction}

Diabetes mellitus is the human disease which results from the presence of high level of blood sugar for prolonged period due to inadequate generation of insulin in blood [1].

In human body, the beta cells in pancreas are responsible for producing the insulin, which regulates the glucose consumption. In diabetes, beta cells fails to produce enough insulin concentration in blood and the human body will be unable to control the blood glucose level.

Type I diabetes mellitus patients cannot produce any insulin and insulin shots are given several times a day to help regulate their blood glucose level. A typical patient is then serving himself as a control system [2]. On the other hand, any patient that suffers from diabetes and not receives the insulin cure properly can lead to complications such as nerve damage, brain damage, amputation and eventually death.

In the human body, the normal blood glucose level varies in a narrow range (70-110) $\mathrm{mg} / \mathrm{dL}$. The diabetes is diagnosed if the human body is not able to control the normal glucose-insulin interaction [3]. For this reason, the blood glucose must be regulated by injecting the insulin [4].

In general, the closed loop glucose regulation system requires three components, which are: glucose sensor, insulin pump and control method for determining the necessary insulin dosage based on the glucose measurements [5]. Figure (1) shows the block diagram of closed loop glucose control system. 


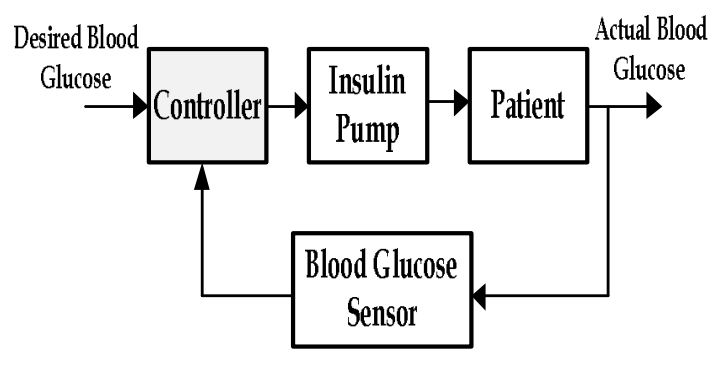

Fig. 1. Block diagram of closed loop insulin regulation system [10].

Several approaches have been previously addressed to design the feedback controller for insulin delivery, such as classical methods like Proportional Integral Derivative (PID) controllers $[6,7]$ and pole placement [8], which require a linearized mathematical model for the design of the controller, as well as model predictive control (MPC) $[9,10]$. In [6] a PID controller based on BP neural networks is proposed in order to reduce the time of lowering blood glucose. In [11], the parameters of Hammerstein controller were optimized in order to minimize the time that takes for blood glucose to come back to its basal level. Also there are some efforts to use model independent based controller such as fuzzy controllers. In [12], a closed-loop control system applying fuzzy logic control introduced and the performance of this controller is tested on three different diabetic patients. Maryam [13] tried to tune the PD fuzzy controller with PSO algorithm. These fuzzy controllers were just able to control the glucose concentration, and suffer from lack of insulin and pump control. The works referred in $[14,15]$ suggested robust controllers such as disturbance rejection LQ controller, $H_{2}$ and $H_{\infty}$ controller to regulate glucose-insulin system for Type I diabetic patients under meal disturbance.

In this paper, four different controllers (optimal LQR, minimax optimal, sliding mode, integral sliding mode) are addressed and designed for the glucose concentration level control problem in diabetic patients under meal disturbance.

\section{Mathematical Model}

Bergman minimal mathematical model, which is the most common referenced model in the literature, approximates the dynamic behavior of a diabetic patient's blood glucose concentration to the insulin injection. The main advantage of using
Bergman minimal model is that the number of parameters is minimum and it describes the relation between main two factors, insulin and glucose concentrations, without getting into biological complicated details. In the present work, nonlinear three-state minimal model of Bergman is considered [7];

$$
\begin{gathered}
\dot{G}(t)=-p_{1} G(t)-X(t)\left(G(t)+G_{B}\right)+h(t) \\
\dot{X}(t)=-p_{2} X(t)+p_{3} Y(t) \\
\dot{Y}(t)=-p_{4}\left(Y(t)+Y_{B}\right)+i(t) / V_{L}
\end{gathered}
$$

Where $G(t)$ is plasma glucose deviation, $[\mathrm{mg} / \mathrm{dL}]$, $X(t)$ is remote compartment insulin utilization, [1/min] and $Y(t)$ is plasma insulin deviation, $[\mathrm{mU} / \mathrm{dL}]$. The control variable $i(t)$ is the exogenous insulin infusion rate $(\mathrm{mU} / \mathrm{min})$, while the disturbance $h(t)$ represents the exogenous glucose infusion rate $(\mathrm{mg} / \mathrm{dL} \mathrm{min})$.

The physical parameters $G_{B}$ and $Y_{B}$ are the basal glucose level $(\mathrm{mg} / \mathrm{dL})$, and basal insulin level $(m U / d L)$, respectively, and $V_{L}$ is the insulin distribution volume $(d L)$. The model parameters are:

$p_{1}(1 / \mathrm{min}), \quad p_{2}(1 / \mathrm{min}), \quad p_{3}\left(d L /\left(m U \min ^{2}\right)\right)$ and $p_{4}(1 / \mathrm{min})$.

If the unmeasurable variable $X(t)$ is assumed a slow variable, then $\dot{X}(t)=0$. From Eq.(1), the expression $X(t)=\left(p_{3} / p_{2}\right) Y(t)$ can be found. Substitution this expression into the first equation, the model of Eq.(1) is reduced to the following [13]:

$$
\begin{aligned}
& \dot{G}(t)=-p_{1} G(t)-\frac{p_{3}}{p_{2}} Y(t)\left(G(t)+G_{B}\right)+h(t) \\
& \dot{Y}(t)=-p_{4}\left(Y(t)+Y_{B}\right)+i(t) / V_{L}
\end{aligned}
$$

The linearization of Eq.(2) is performed by taking the variation of $G(t)=G_{o}+\Delta G(t)$ and $Y(t)=$ $Y_{o}+\Delta Y(t)$ around equilibrium points $\left(G_{o}, Y_{0}, h_{o}\right.$, $i_{o}$ ). The perturbed version of Eq.(2) is given by;

$$
\begin{aligned}
\Delta \dot{G}= & \left(-p_{1}-\frac{p_{3}}{p_{2}} Y_{o}\right) \Delta G(t) \\
- & \frac{\left(G_{0}+G_{B}\right) p_{3}}{p_{2}} \Delta Y(t) \\
\Delta \dot{Y}(t)= & -p_{4} \Delta Y(t)+\Delta i(t) / V_{L}
\end{aligned}
$$

If $\Delta G(t)$ is defined as the first state variable $x_{1}(t)$, $\Delta Y(t)$ is set as second state variable $x_{2}(t)$ and $u(t)$ is assigned to control input variation $\Delta i(t)$, then the previous equation can be written in the following state space form,

$$
\begin{aligned}
& \dot{\boldsymbol{x}}(t)=\left[\begin{array}{cc}
-p_{1}-\frac{p_{3} Y_{o}}{p_{2}} & -\frac{\left(G_{0}+G_{B}\right) p_{3}}{p_{2}} \\
0 & -p_{4}
\end{array}\right] \boldsymbol{x}(t) \\
& +\left[\begin{array}{c}
0 \\
1 / V_{L}
\end{array}\right] u(t)+\left[\begin{array}{l}
1 \\
0
\end{array}\right] h(t) \\
& \boldsymbol{y}(t)=\left[\begin{array}{ll}
1 & 0 \\
0 & 1
\end{array}\right] \boldsymbol{x}(t)
\end{aligned}
$$


For control objectives, the linearization in state space of the above model is taken at the equilibrium points of the following specified values;

$h_{o}=0, i_{o}=p_{4} Y_{B} V_{L}, G_{o}=0$

Therefore, the obtained linearized model can be written as;

$\dot{x}(t)=\left[\begin{array}{cc}-p_{1} & -\frac{G_{B} p_{3}}{p_{2}} \\ 0 & -p_{4}\end{array}\right] x(t)+\left[\begin{array}{cc}1 & 0 \\ 01 / V_{L}\end{array}\right] u_{t}(t)$

$y(t)=\left[\begin{array}{ll}1 & 0 \\ 0 & 1\end{array}\right] x(t)+$

where $u_{t}(t)=\left[\begin{array}{ll}h(t) & u(t)\end{array}\right]^{T}$. Equation(5) can be written in compact form as;

$\dot{x}(t)=\boldsymbol{A} x(t)+\boldsymbol{B} u(t)$

$y(t)=\boldsymbol{C} x(t)$

It is easily to show that the linearized model is completely controllable.

\section{Controller Design}

Four structures of advanced controllers will be presented and designed here for controlling the glucose level in human blood under meal disturbance. Later, the performance of such controllers will be verified and compared to each other using Matlab/Sumlink.

\subsection{Sliding Mode Controller}

Sliding mode control is a discontinuous feedback control forces the system states to reach and remain on a specific surface within the state space (called sliding surface).

The first stage of design is the selection of the discontinuity surface such that sliding motion would exhibit desired properties.

Let us define a surface $s$ in the state space as follows $[16,17]$;

$s=x_{2}+c x_{1}$

If a controller $u$ was designed to make the system trajectories head to the surface $s=0$, then Eq.(7) can be written as,

$x_{2}+c x_{1}=0$

From Eq. (5), one can find that

$\dot{x}_{1}=-p_{1} x_{1}-\left(G_{B} p_{3} / p_{2}\right) x_{2}$

Rearranging the above equation results in

$x_{2}=-p_{2}\left(p_{1} x_{1}+\dot{x}_{1}\right) /\left(G_{B} p_{3}\right)$

Substituting $x_{2}$ from the above equation into Eq.(8) results in

$-p_{2}\left(p_{1} x_{1}+\dot{x}_{1}\right) /\left(G_{B} p_{3}\right)+c x_{1}=0$

or, $\dot{x}_{1}+\left(p_{1}-c G_{B} p_{3} / p_{2}\right) x_{1}=0$

The time solution for the equation above is written as

$x_{1}=x_{1}(0) e^{-\left(p_{1}-c G_{B} p_{3} / p_{2}\right) t}$

such that $\left(p_{1}-c G_{B} p_{3} / p_{2}\right)>0$.

Equation (13) shows that if the state trajectories are forced to move on surface $s=0$, then $x_{1}$ will tend to zero exponentially after a finite time interval or one can say,

$x_{1}(t=\infty)=0 \rightarrow G(t)=0$

To ensure that the state trajectories will head toward the surface $s=0$, the following reaching condition should be fulfilled

$s \dot{S}<0$

Since $\dot{s}=\dot{x}_{2}+c \dot{x}_{1}$, then

$$
\begin{gathered}
s \dot{s}=s\left[\dot{x}_{2}+c \dot{x}_{1}\right] \\
=s\left[-p_{4} x_{2}+u(t) / V_{L}-p_{1} x_{1}\right. \\
\left.-\left(p_{3} / p_{2}\right) x_{1}+h(t)\right]
\end{gathered}
$$

If one assumes that the control defined as a discontinuous function for the surface $s$ as below $u(t)=-k * \operatorname{sign}(s)$

Substituting for the controlu(t) in Eq.(16), we have

$s \dot{s}=-p_{4} s x_{2}-k s \operatorname{sign}(s) / V_{L}$

$-p_{1} s x_{1}-\left(p_{3} / p_{2}\right) s x_{1}+s h(t)$

Using the fact that $s * \operatorname{sign}(s)=|s|$ and from linear algebra, the inequality $a b \leq|a||b|$ holds and results in the following;

$s \dot{s} \leq|s|\left\{\left|p_{4}\right|\left|x_{2}\right|-k / V_{L}+\left|p_{1}\right|\left|x_{1}\right|\right.$

$\left.+\left|p_{3} / p_{2}\right| x_{1}+|h(t)|\right\}<0$

Solving for $k$, we have

$k>V_{L}\left\{\left|p_{4}\right|\left|x_{2}\right|+\left|p_{1}\right|\left|x_{1}\right|+\left|p_{3} / p_{2}\right| x_{1}\right.$

$\left.+|h(t)|_{\max }\right\}$

\subsection{Integral Sliding Mode Control for Disturbance Rejection}

In what follows, integral sliding mode controller is designed for control and disturbance rejection of glucose systems. Starting with rewriting the control law as follows,

$u=u_{0}+u_{1}$

where $u_{0}$ is designed to make the system follows a specified trajectory $x_{0}$ in the state space which is based on optimal LQR controller, while the design of $u_{1}$ is dedicated to cancel the disturbance $h(t)$.

Rewriting Eq.(4) by separating the control input $i(t)$ from disturbance $h(t)$ and then substituting the control signal from Eq.(21) to have;

$\dot{\boldsymbol{x}}=\boldsymbol{A} x(t)+\boldsymbol{B}_{u} u_{0}(t)+\boldsymbol{B}_{u} u_{1}(t)+\boldsymbol{L} h(t)$

Where $\boldsymbol{L}=\left[\begin{array}{ll}1 & 0\end{array}\right]^{T}$ and $\boldsymbol{B}_{u}=\left[\begin{array}{ll}0 & 1 / V_{L}\end{array}\right]^{T}$. 
The first stage of design is to select the discontinuity surface $s$ such that sliding motion would exhibit desired properties. The surface $s$ is defined in the state space as follows

$s=s_{0}+z$

Wherez represents the integral part which cancels the disturbance and $s_{0}$ represent the desired path in the state space given by

$s_{0}=\boldsymbol{C} x$

In order to keep the system on the specified trajectory $s_{0}$ the time derivative of the surface $s$ should equal zero. In other words, the system trajectory will not leave the surface $s_{0}$.

$\dot{s}=\dot{s}_{0}+\dot{z}=0$

$=\boldsymbol{C} \dot{x}+\dot{z}=0$

or,

$$
\begin{gathered}
\dot{s}=\boldsymbol{C}\left[\boldsymbol{A} x(t)+\boldsymbol{B}_{u} u_{0}(t)+\boldsymbol{B}_{u} u_{1}(t)+\right. \\
\boldsymbol{L} h(t)]+\dot{z}=0
\end{gathered}
$$

In order to ensure that $s(t)=s_{0}(t)$ for all $t>0$

the following condition should be

fulfilled $\boldsymbol{B}_{u} u_{1}(t)=-\boldsymbol{L} h(t)$

Substitute in $\dot{s}$ we get

$\dot{z}=-\boldsymbol{C}\left[\boldsymbol{A} x(t)+\boldsymbol{B}_{u} u_{0}(t)\right]$

or,

$z=-\boldsymbol{C} \int_{t_{0}}^{t}\left[\boldsymbol{A} \boldsymbol{x}(t)+\boldsymbol{B}_{u} u_{0}(t)\right] d t$

Thus, substitution for $z$ is the surface equation $s$ will ensure that the system trajectories will remain on the surface $s$ even under external disturbances. If $u_{1}$ considered to be a nonlinear function, then

$u_{1}=-k_{1} \operatorname{sign}(s)$

where $k_{1}$ is the discontinuous controller gain. The final control law can be rewritten as follows;

$u=u_{0}+u_{1}$

$u=-K x-k_{1} \operatorname{sign}(s)$

To ensure that $\dot{s}=0$ for $\forall t \geq 0$ in Eq.(25), the following condition has to be satisfied

$\boldsymbol{C B}_{u} u_{1}(t)=-\boldsymbol{C} \boldsymbol{L} h(t)$

Multiplying out the matrices in the above equation results in,

$-k_{1} \operatorname{sign}(s) / V_{L}=h(t)$

Taking the worst case disturbance $h(t)_{\max }$, the integral sliding mode gain $k_{1}$ can be evaluated as follows

$k_{1} \geq V_{L} h(t)_{\max }$

\subsection{Optimal LQR Control}

The requirement of classical LQ control method is to minimize the following quadratic cost functional:

$J(u(t))=\frac{1}{2} \int_{0}^{\infty}\left\{y^{T} \boldsymbol{Q} y+u^{T} \boldsymbol{R} u\right\} d t$

The classical LQ attempts to find an optimal $u^{*}(t), t \in[0, \infty]$ such that $J\left(u^{*}(t)\right) \leq J(u(t)$ for all $u(t), t \in[0, \infty]$ under properly chosen $R$ and $\boldsymbol{Q}$. The matrix $\boldsymbol{Q}(n \times n)$ and $\boldsymbol{R}(m \times m)$ are selected by the design engineer.

The first element of input vector is the insulin rate $i(t)$, and the second element is the exogenous glucose $h(t)$ which stands for disturbance. The objective is to minimize the effect of disturbance on the output. However, the elements of diagonal matrix $R$ are responsible for overweighting and underweighting of the input elements. The first component of matrix $R$, namely $R_{11}$, is chosen to give high importance to disturbance input, $h(t)$, while the element $R_{22}$ has to giveless importanceto insulin rate. Less importance to insulin means that we relief the constraint on insulin or permit it to take higher level. Therefore, high value is assigned to $R_{11}$ and low value is assigned to $R_{22}$. On the other hand, the elements of $\boldsymbol{Q}$ give the weights on system outputs, plasma glucose deviation and plasma insulin deviation. Normally in this application, the same weight is given to both outputs.

Based on optimal control theory, the following control Riccatiequation is established as [18],

$\boldsymbol{P A}+\boldsymbol{A}^{T} \boldsymbol{P}+\boldsymbol{C}^{T} \boldsymbol{C}-\boldsymbol{P} \boldsymbol{B}^{T} \boldsymbol{R}^{-1} \boldsymbol{B} \boldsymbol{P}=[\mathbf{0}] \quad \ldots$ (35) Since Matlab package is used here, a special builtin function called care is used to solve above Ricatti equation for matrix $\boldsymbol{P}$. Positive definiteness of matrix $\boldsymbol{P}$ is a necessary condition of solution. The optimal solution can be found in terms of $\boldsymbol{P}$-matrix as follows;

$u^{*}(t)=-\boldsymbol{R}^{-1} \boldsymbol{B}^{T} \boldsymbol{P} \boldsymbol{x}^{*}(t)$

The closed-loop system based on optimal control input is given by [18];

$\dot{\boldsymbol{x}}^{*}(t)=[\boldsymbol{A}-\boldsymbol{B} \boldsymbol{K}] \boldsymbol{x}^{*}(t)$

where $\boldsymbol{K}=\boldsymbol{R}^{-1} \boldsymbol{B}^{T} \boldsymbol{P}$.

The closed-loop system will exhibit different performance depending on how design parameters are selected. Generally speaking, selecting $\boldsymbol{Q}$ with large terms means that, to keep Jsmall, the state $\boldsymbol{x}(t)$ must be smaller. On the other hand selecting $\boldsymbol{R}$ with large terms means that the control input $u(t)$ must be smaller to keep $J$ small. This means that larger values of $\boldsymbol{Q}$ generally result in the closed loop poles of the system matrix $(\boldsymbol{A}-\boldsymbol{B} \boldsymbol{K})$ being further left in the s-plane so that the state decays faster to zero. On the other hand, larger $R$ means that less control effort is used, so that the poles are generally slower, resulting in larger values of the state $\boldsymbol{x}(t)$. 


\subsection{Optimal LQR Control with Disturbance Rejection (Minimax)}

The disturbance rejection LQ method is a generalization of classical LQ method and is based on the minimax criteria. This techniques is dedicated for optimal control and disturbance rejection puposes. Figure (2) shows the general structure of disturbance rejection LQ (minmax) control strategy.

The system dynamic given by Eq.(4) is reformulated by separating the control input $i(t)$ from disturbance $h(t)$. Therefore, the dynamic system can be written as;

$$
\begin{aligned}
& \dot{x}=\boldsymbol{A} x(t)+\boldsymbol{B}_{u} i(t)+\boldsymbol{L} h(t) \\
& y(t)=\boldsymbol{C} x(t)+\boldsymbol{D} u \\
& \text { where } \boldsymbol{B}_{u}=\left[\begin{array}{ll}
1 & 1 / V_{L}
\end{array}\right]^{T} \text { and } \boldsymbol{L}=\left[\begin{array}{ll}
1 & 0
\end{array}\right]^{T} .
\end{aligned}
$$

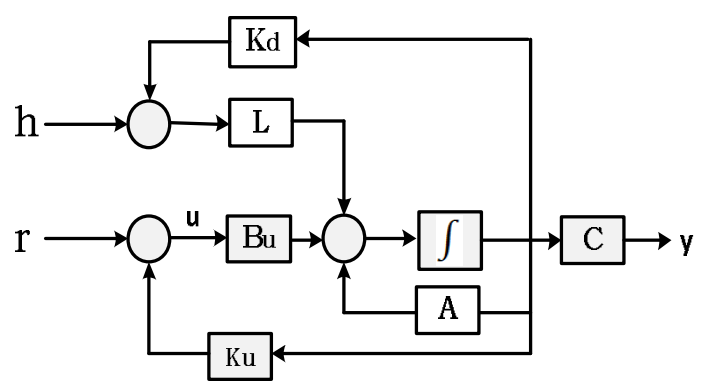

Fig. 2.General structure of Minimax Control [15].

In this case, the quadratic cost functional will be modified with the disturbance explicitly

$J(i(t))=\frac{1}{2} \int_{0}^{\infty}\left\{y^{T}(t) y(t)+i^{T}(t) i(t)\right.$

where $\gamma$ is a design parameter, which is concerned in system stability analysis.

It is clear that the disturbance tries to maximize the cost, so it appears with negative sign. Meanwhile, the objective is to find a control $i(t)$ that could minimize the maximum cost achievable by the disturbance. The unique solution of cost function, $i^{*}(t)$ and $h^{*}(t)$, exists and satisfies the saddle point condition,

$J\left(i^{*}(t), h(t)\right) \leq J\left(i(t), h^{*}(t)\right)$

$\leq J\left(i^{*}(t), h^{*}(t)\right)$

where $i^{*}(t)$ is the optimal control and $h^{*}(t)$ is the worst-case disturbance. These functions can be computed as:

$i^{*}(t)=-\boldsymbol{B}_{u}^{T} \boldsymbol{P} \boldsymbol{x}(t)$

$h^{*}(t)=\left(1 / \gamma^{2}\right) \boldsymbol{L}^{T} x(t)$

where $\boldsymbol{P}$ is positive symmetric matrix. The numeric values of matrix entries are determined by the solution of the following Modified Control Riccati Equation (MCRE);

$$
\boldsymbol{P A}+\boldsymbol{A}^{T} \boldsymbol{P}+\boldsymbol{C}^{T} \boldsymbol{C}-\boldsymbol{P}\left(\boldsymbol{B}_{u}^{T} \boldsymbol{B}_{u}-\boldsymbol{L L}^{T}\right) \boldsymbol{P}=[0]
$$

To solve Riccatiequation using Matlab built-in CARE function, the structure of above Riccati equation is modified to be rewritten as

$$
\begin{aligned}
& \boldsymbol{P A}+\boldsymbol{A}^{T}-\boldsymbol{P}\left[\boldsymbol{L} \boldsymbol{B}_{u}\right]\left[\begin{array}{cc}
-\gamma^{2} & 0 \\
0 & 1
\end{array}\right]^{-1}\left[\boldsymbol{L}^{T} \boldsymbol{B}_{u}^{T}\right] \boldsymbol{P} \\
& +\boldsymbol{C}^{T} \boldsymbol{C}=[0] \\
& \text { where, } \boldsymbol{R}=\left[\begin{array}{cc}
-\gamma^{2} & 0 \\
0 & 1
\end{array}\right] \text { and } \mathbf{B}=\left[\boldsymbol{L} \boldsymbol{B}_{u}\right] .
\end{aligned}
$$

The RE described by Eq.(43) finds a straightforward solution with the help of CARE function as indicated in Appendix (A).

\section{Simulation Results}

Both sliding mode and optimal LQR controllers have been applied to the diabetic model. The effectiveness and robustness of suggested controllers are assessed using simulation results based on Matlab-Simulink (Ra2012).

Figure (3) shows meal disturbance function behavior, which represents the exogenous glucose infusion. Figure (4) shows the Simulink modeling of sliding and optimal controllers for controlling the glucose level against disturbing meal. The meal disturbance is saved inside a look-up table (see Appendix B).

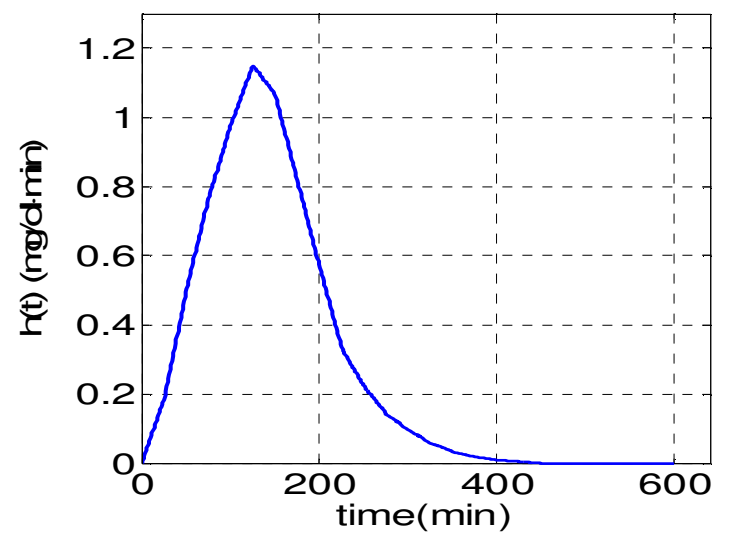

Fig. 3. Disturbance meal function (exogenous glucose infusion). 


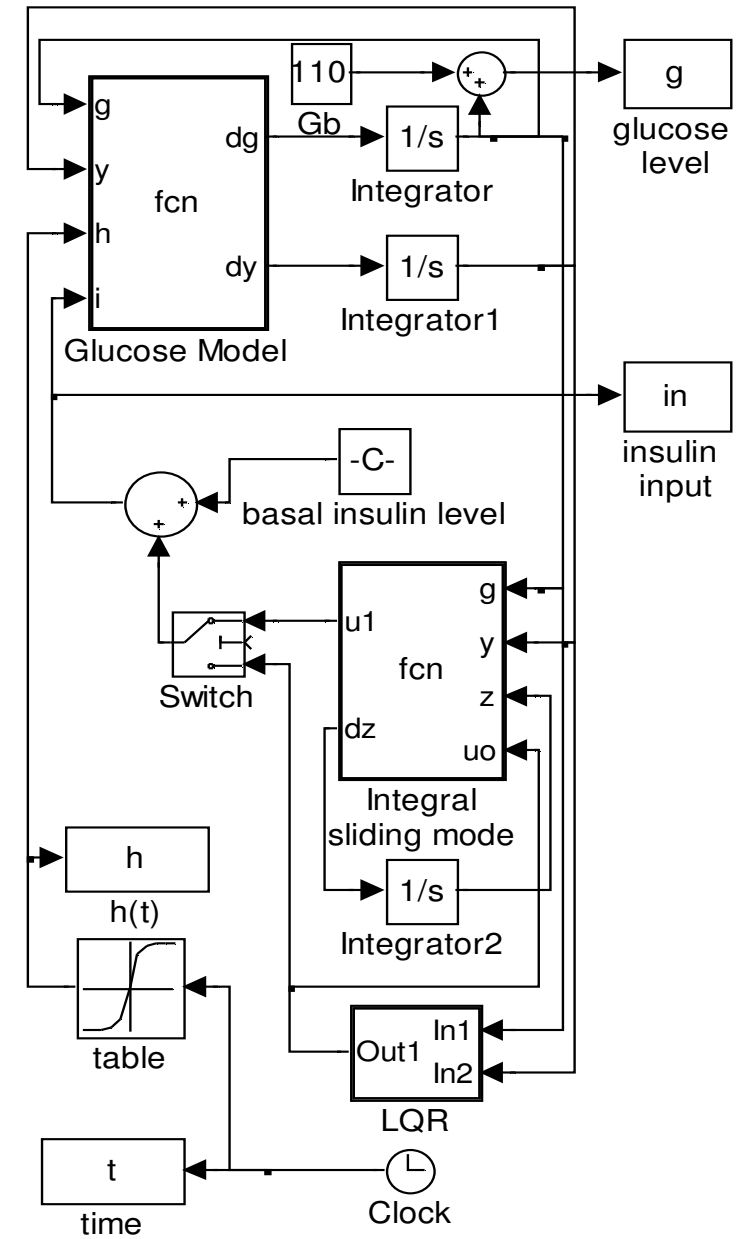

Fig .4. Matlab-Simulink Modeling of Sliding Mode Controller and Optimal Controllers.

The model parameters are listed below [15]: $p_{1}=0.028, p_{2}=0.025, p_{3}=0.00013$, $p_{4}=0.093, G_{b}=110, Y_{b}=1.5, i_{0}=16.67$.

Based on the above values, the design parameter $\boldsymbol{K}$ is calculated using Eq.(13). Then, the value of $\boldsymbol{K}$ is equal -2.23 .

For the optimal LQR controller, the matrices $\boldsymbol{R}$ and $\boldsymbol{Q}$ are set at the following values;

$\boldsymbol{R}=\left[\begin{array}{cc}1 & 0 \\ 0 & 1 / 1000\end{array}\right], \mathbf{Q}=\left[\begin{array}{cc}1 / 1000 & 0 \\ 0 & 1 / 1000\end{array}\right]$,

Considering the above

$\boldsymbol{P}=\left[\begin{array}{cc}0.0140 & -0.0560 \\ -0.0560 & 0.3130\end{array}\right]$

Then, it is easily to find the optimal gain matrix $\boldsymbol{K}$ $\boldsymbol{K}=\left[\begin{array}{cc}0.0000 & -0.0001 \\ -0.4664 & 2.6086\end{array}\right]$

Based on critical value of $\gamma=17.086$, which lead to positive definite matrix solution and stable closed loop system, the matrix $P$ resulting from Eq.(31) is given by

$\boldsymbol{P}=\left[\begin{array}{cc}3.5822 & -3.2982 \\ -3.2982 & 7.02982\end{array}\right]$

This gives the following feedback gain matrix
$\boldsymbol{K}=\left[\begin{array}{cc}0.0123 & -0.0113 \\ -27.4853 & 58.5570\end{array}\right]$

To determine the stability of the system one can easily substitute the values of gain matrix Kinto the closed loop equation represented by Eq.(5) and find the location of the closed loop as in the following manner:

$\dot{\boldsymbol{x}}=(\boldsymbol{A}-\boldsymbol{B} \boldsymbol{K}) \boldsymbol{x}$

Then, the eigenvalues of the matrix $(\boldsymbol{A}-\boldsymbol{B} \boldsymbol{K})$ are the roots of the characteristic equation which calculated as follows [18]

$|\lambda \boldsymbol{I}-\boldsymbol{A}+\boldsymbol{B} \boldsymbol{K}|$

$=\mid\left[\begin{array}{ll}\lambda & 0 \\ 0 & \lambda\end{array}\right]-\left[\begin{array}{cc}-0.0280 & -0.572 \\ 0 & -0.093\end{array}\right]$

$+\left[\begin{array}{cc}1 & 0 \\ 0 & 1 / 120\end{array}\right]\left[\begin{array}{cc}0 & 0 \\ -0.4664 & 2.6086\end{array}\right]$

$=\mid\left[\begin{array}{cc}\lambda+0.0280 & -0.572 \\ 0 & \lambda+0.093\end{array}\right]$

$+\left[\begin{array}{cc}0 & 0 \\ -0.0039 & 0.2174\end{array}\right] \mid$
$=\left|\left[\begin{array}{cc}\lambda+0.0280 & -0.572 \\ -0.0039 & \lambda+0.3104\end{array}\right]\right|$

Solving for $\lambda$ yields

$\lambda_{1}=-0.361, \lambda_{2}=-3.023$

Repeating the same procedure for the optimal Minimax controller we get

$\lambda_{1}=-0.503, \lambda_{2}=-5.587$

Initially, both controllers have the roots in the left hand of the s-plane meaning that the system under the proposed controllers is stable. However, it can be noted that with the optimal Minimax controller the roots lie further from the origin resulting in more stable performance and a faster response.

Figure (5) shows the glucose level under meal disturbance of Figure (2) with different four controllers. The figure shows that the glucose level reaches $136(\mathrm{mg} / \mathrm{dL})$ when using classical optimal LQR controller. This stands for $12.36 \%$ above basal glucose level. However, Minimax optimal controller could reduce the maximum value of glucose level to reach to $125(\mathrm{mg} / \mathrm{dL})$, which is about $11.36 \%$ above the basal level. Sliding mode controller (SMC) gives better performance than both optimal controllers. With this controller, the maximum glucose level does not exceed $123(\mathrm{mg} / \mathrm{dL})$; i.e, the controller permits $11.18 \%$ change over the basal glucose level. It is evident from the figure that integral sliding mode controller shows the best robustness characteristics than all the above controllers. The percentage change of ISMC is approximately $10.63 \%$ above the basal level which is the minimum percentage than others. 


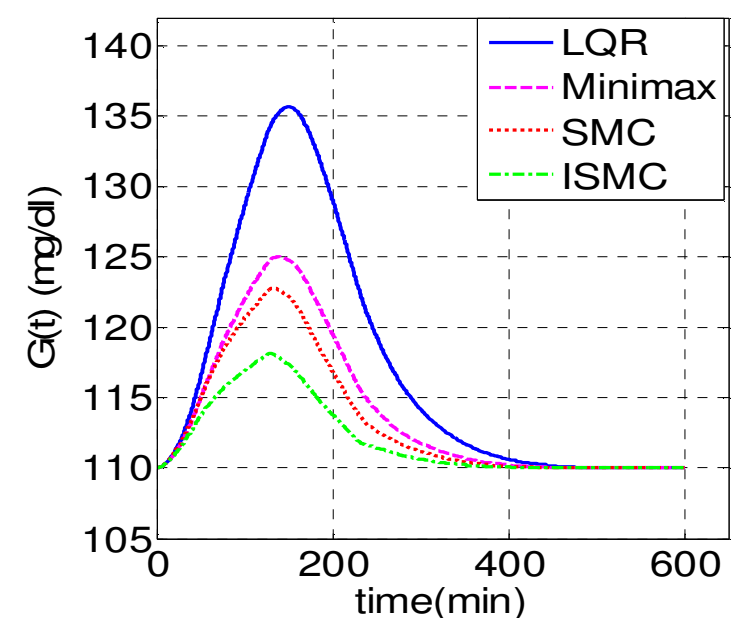

Fig. 5. Plasma glucose level

Figure (6) shows the insulin rate resulting from each controller. It has been shown that the more robust controller, the higher level of insulin rate which is taken by human body. This physically indicates that more robust controller of requires more insulin rate to be injected; this is the price of robustness.

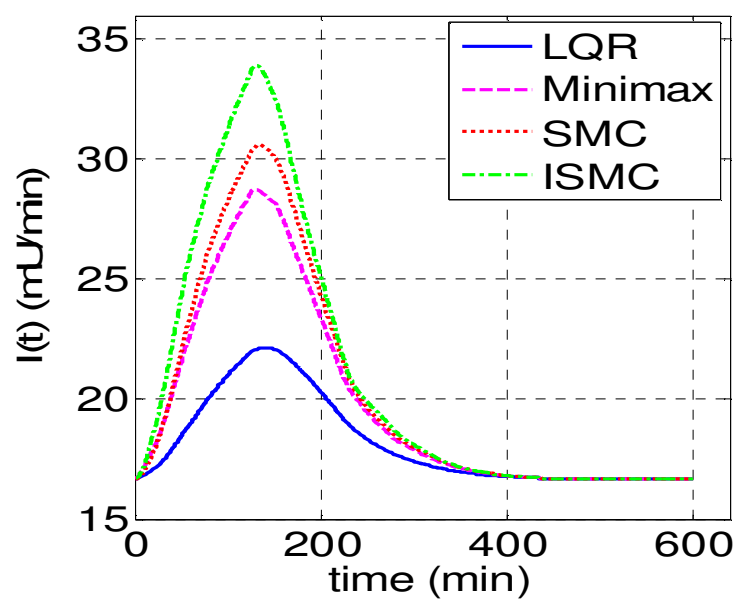

Fig. 6. Plasma glucose level.

\section{Conclusion}

In this paper, different controllers were addressed for the problem of blood glucose concentration level control. Simulation was performed using MATLAB/Simulink in order to investigate the performance of suggested proposed controllers under a meal disturbance. The four designed controllers could successfully control the glucose level successfully and retain the glucose level back to its basal level. However, the simulation has showed relative differences in the performance of addressed controllers.

It can be noted that the integral sliding mode gives the best disturbance rejection capability over other controllers such that it prevents the glucose level to exceed $118 \mathrm{mg} / \mathrm{dl}$. Moreover, sliding mode controller outperforms the characteristics of Minmax controllers. The latter could keep the glucose level within $125 \mathrm{mg} / \mathrm{dl}$. However, it can be seen that the optimal LQR controller have the worst performance where the glucose level reaches the value of $135 \mathrm{mg} / \mathrm{dl}$.

From Fig. (6), it can be concluded the price of getting better control performance is the increased level of insulin infusion in order to counteract the glucose evolution. Therefore, it can be noted that the integral sliding mode has the highest insulin infusion compared to the other controllers, while the lowest level is shown in LQR controller.

\section{References}

[1]Bergman R.N., Philips L.S., Cobelli C., "Physiologic Evaluation of Factors Controlling Glucose Tolerance in Man, Journal of Clinical Investigation," Vol.68, pp. 1456-1467, 1981.

[2] O. Vega-Hernandez, D. U. Campos-Delgado, and D. R. Espinoza Trejo, "Increasing Security in an Artificial Pancreas: Diagnosis of Actuator Faults," Proc. of Pan-American Health Care Exchanges Conference, Mexico City, Mexico, pp. 137-142, 2009.

[3] V. R. Kondepati and H. M. Heise, "Recent Progress in Analytical Instrumentation for Glycemic Control in Diabetic and Critically Ill Patients, "Anal Bional.Chem., Vol. 38,pp. 545563, 2007.

[4] Thomson, S., D. Beaven, M. Jamieson, S. Snively, A.Howl and A. Christophersen, "Type II diabetes: Managing for Better Health Outcomes," in PriceWater House Coopers Report, Diabetes New Zealand Inc., 2001.

[5] Topp, B., K. Promislow and G. De Vries, "A Model of $\beta$-cell Mass, Insulin and Glucose kinetics, "Pathways to diabetes. J. Theor. Bio, pp.605-619, 2000.

[6]C. Li and R. Hu, "PID Control Based on BP Neural Network for the Regulation of Blood Glucose Level in Diabetes", in Proceeding of the 7th IEEE International Conference on Bioinformatics and Bioengineering, Boston, pp. 1168-1172, 2007.

[7] Chee F, Fernando TL, Savkin AV, Heeden VV. "Expert PID Control System for Blood 
Glucose Control in Critically Ill Patients," IEEE Transactions on Information Technology in Biomedicine, pp.419- 425, 2003.

[8] Salzsieder E, Albrecht G, Fischer U, Freyse EJ. "Kinetic Modeling of the Glucoregulatory System to Improve Insulin Therapy," IEEE Transactions on Biomedical Engineering, Vol.32, pp.846-855, 1985.

[9]Parker R.S., Doyle F.J., Peppas NA., "A Model-based Algorithm for Blood Glucose Control in Type I Diabetic Patients," IEEE Transactions on Biomedical Engineering, Vol.46, No.2, pp.148-157, 1999.

[10] S. M. Lynch and B. W. Bequette,"Model predictive Control of Blood Glucose in Type I diabetes Using Subcutaneous Glucose Measurements, "in Proceedings of the American Control Conference, U.S.A, pp. 4039-4040, May 2002.

[11] K. Beyki, M. Dashti Javan, K. Shojaee and M.M. Neshati, "An Intelligent Approach for Optimal Regulation of Blood Glucose Level," In 17th Iranian Conference of Biomedical Engineering, pp.1-5, 2010.

[12] S. Yasini, M.B. Naghibi-Sistani and A. Karimpour, "Active Insulin Infusion Using Fuzzy-based Closed-loop Control", in 3rd International Conference on Intelligent System and Knowledge Engineering, Mashhad, Iran, pp. 429-434, 2008.

[13] D. N. Maryam Abadi, A. Alfi, M. Siahi, "An Improved Fuzzy PI Controller for Type 1 Diabetes," Research Journal of Applied Sciences, Engineering and Technology, Vol.4, No.21, pp. 4417-4422, 2012.

[14] Levente Kovacs, Béla Palancz, Endre Borbely, "Robust Control Algorithm for Blood Glucose Control Using Mathematica," Acta Electrotechnicaet Informatica, Vol. 10, No. 2, pp. 10-15, 2010.

[15] Levente Kovacs, Béla Palancz, "GlucoseInsulin Control of Type1 Diabetic Patients in $\mathrm{H}_{2} / \mathrm{H}_{\infty}$ Space via Computer Algebra," 2nd International Conference, Australia, July, 2007.

[16] Vadim Utkin, Jürgen Guldner, Jingxin Shi, "Sliding Mode Control in ElectroMechanical Systems," CRC Press, 2009.
[17] Jinkun Liu, Xinhua Wang, "Control for Mechanical Systems Design, Analysis and MATLAB Simulation," Tsinghua University Press, Beijing and Springer-Verlag Berlin Heidelberg, 2012.

[18] Donald E. Kirk, "Optimal Control Theory: Introduction, "Dover Publications, Inc., 1998.

\section{Appendix (A)}

Matlab function of solution of Equation (12), using CARE function;

gama $=17.086$;

$\mathrm{C}=\left[\begin{array}{ll}1 & 0\end{array}\right]$;

$\mathrm{Q}=\mathrm{C}^{\prime} \mathrm{C}$

$\mathrm{L}=[1 ; 0]$;

$\mathrm{Bu}=[0 ; 1 / 120]$;

$\mathrm{B}=\left[\begin{array}{ll}\mathrm{L} & \mathrm{Bu}\end{array}\right]$;

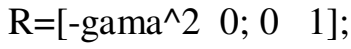

$\mathrm{Pm}=\operatorname{care}(\mathrm{A}, \mathrm{B}, \mathrm{Q}, \mathrm{R})$

$\mathrm{Km}=\operatorname{inv}(\mathrm{R}) * \mathrm{~B}^{\prime} * \mathrm{Pm}$

\section{Appendix (B)}

The Table below lists the data of disturbing meal behavior

Table 1,

Disturbance meal function

\begin{tabular}{ll}
\hline $\begin{array}{c}\text { Time (t) } \\
\text { [min] }\end{array}$ & $\begin{array}{l}\text { Glucose }(\mathbf{h}(\mathbf{t})) \\
{[\mathbf{m g} /(\mathbf{d L} \cdot \mathbf{m i n}]}\end{array}$ \\
\hline 0 & 0 \\
25 & 0.185 \\
50 & 0.495 \\
75 & 0.765 \\
100 & 0.975 \\
125 & 1.15 \\
150 & 1.07 \\
175 & 0.82 \\
200 & 0.575 \\
225 & 0.335 \\
250 & 0.225 \\
275 & 0.145 \\
300 & 0.098 \\
325 & 0.06 \\
350 & 0.035 \\
375 & 0.02 \\
400 & 0.01 \\
450 & 0.005 \\
1000 & 0 \\
\hline
\end{tabular}




\title{
مقارنة الاداء بين مسيطرات متقدمة للسيطرة على مستوى السكر في الجسم البشري تحت تاثير الاضطر اب الغذائي
}

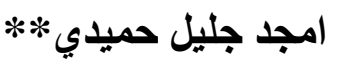

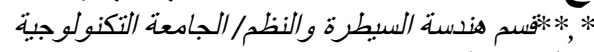

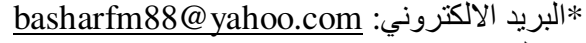 \\ aaaacontrol2010@yahoo.com البريد الالكتروني: الاكتي: *
}

\section{الخلاصة}

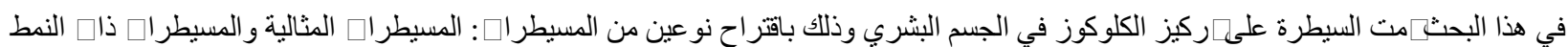

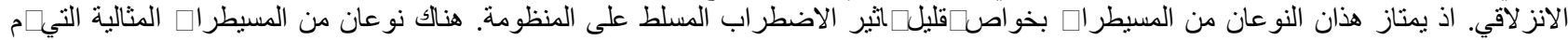

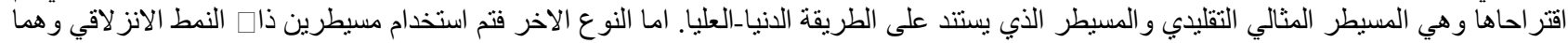
المسيطر التقليدي والتكاملي التيطر التئي.

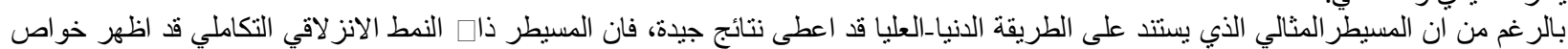

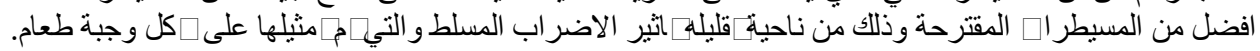

